\section{Scientific communication: Writing up}

\section{In the competitive world of scientific publishing, it is essential to communicate research findings in a clear and accessible manner. Scientists should develop the ability to write well-structured and compelling cover letters, manuscripts and rebuttal letters.}

Writing a manuscript involves compressing months or years of work, often performed by dozens of hands, into a single document. It can be a trying exercise, but concise and clear communication is important to effectively and efficiently share your data with the scientific community.

Manuscripts submitted to Nature Cell Biology are first read by the primary handling editor and then discussed within the editorial team. We also pay close attention to the cover letter. The decision to send a manuscript for peer review is never a factor of the style or clarity of writing, but is instead determined by the advance provided for a broad cell biology audience in the context of the published literature (see also our previous editorial on peer review). However, a lucid and well-organized manuscript, accompanied by a coherent cover letter, can help convey the key elements of the research to the editors and external reviewers.

A cover letter is the ideal place to communicate the novelty and potential interest of the dataset. Although the editor will assess the study's advance in light of the published literature, the author's perspective on how the findings fit within the context of the broader literature is also helpful. Therefore, a strong and cogent statement about how the study advances the field can enhance the overall presentation of a manuscript: over-interpreting or overhyping the data will not. The cover letter should also note whether related research, generated by the authors' own lab or by competitors, is under consideration at another journal. Finally, suggestions for potential referees or reviewers to exclude can also be listed.

The manuscript text and figures should conform to the format guidelines in our 'Guide to Authors'. Before submission, authors might wish to solicit feedback from colleagues and collaborators, or enlist the services of a company that performs professional scientific editing (for example, MSC Scientific Editing), to ensure that the manuscript is clear and accessible, and that the data are interpreted in a balanced manner. Making figures can drive the non-computer-savvy - and even Adobe Illustrator savants - to the brink, but creating figures does not require advanced coursework in graphic design. Bang Wong, in his fascinating columns in Nature Methods, provides excellent suggestions for figure layout, colour combinations, and even more esoteric issues such as typography and the judicious use of arrows. In particular, in fairness to readers with colour blindness, we ask that red-green colour combinations be avoided when possible. Figures must be of sufficient resolution so that the editors, and potentially peer reviewers, can make sense of the data. If figure quality has suffered during online submission, higherresolution figures can be provided electronically or by post (as CDs/ DVDs or glossy prints). We will ensure that the material is distributed to the referees. Data should not be manipulated or 'beautified' in a way that obscures or misrepresents the findings (see our previous editorial on data beautification and fraud and refer to our 'Guide to Authors' for current guidelines on image integrity).

You've submitted your cover letter and manuscript, received encouraging reports from the referees, and made the appropriate revisions: now what? A rebuttal letter that provides a concise point-by-point response to referees' comments is essential. The rebuttal should outline precisely how the manuscript was revised to address the referees' concerns and guide them to the specific figure, text section or supplementary information item that was revised. Reviewing manuscripts requires considerable effort and time, and a coherent and detailed rebuttal letter can greatly assist referees in their task. Long-winded, opaque or vitriolic rebuttals carry the risk of antagonizing referees. For example, a letter that consolidates responses to similar issues raised by different referees reduces the clarity of the rebuttal, and makes efficient review of a revised manuscript that much more difficult. In some cases, we will ask an author to rewrite his or her rebuttal letter if it is deemed to be completely unsuitable. The accompanying cover letter, which is not shared with the referees, should provide an overview of the revisions and highlight any major changes in the overall conclusions. Once again, details of any related work under consideration elsewhere should be provided.

After a manuscript is accepted for publication, the production editors ensure that the text and figures comply with journal style, but ultimately the responsibility for scientific communication rests with the authors. Writing clearly and concisely can help to convey the central aspects of a study and ensure that the results are accessible to specialists and non-specialists alike.

\section{NCB tweets}

\section{Nature Cell Biology editors highlight research and news relevant to cell and developmental biologists.}

As this issue of the journal went to press, we started tweeting. We hope to draw attention to recent research published in this journal (such as new content published online, special projects and conference attendance by editors) as well as research from across Nature Publishing Group and beyond. In addition, we will also tweet news items of interest to our readers, including new developments in science policy and funding, issues affecting graduate students and postdocs, and other topics that are featured in our editorial pages. Follow us @naturecellbio and do join in the conversation. 